\title{
310 - Enhancing communication between nursing staff and people with dementia, their informal carers and other involved professionals: development of tools
}

Claudia van der Velden, Marjolein Veerbeek, Bernadette Willemse

Background Nursing staff play an important role in the care of people with dementia (pwd), both at home and in residential care. Communication is essential in providing person centered care to people with dementia. However, symptoms of the disease, such as deterioration in memory, concentration, thought and speech, make communication with pwd more challenging. Therefore, at the request of nursing staff, we started to developed tools to support nursing staff to better communicate with pwd.

Methods First, we identified bottlenecks in communication experienced by nursing staff, informal carers and pwd both through online questionnaires among nursing staff $(n=1.070)$ and informal caregivers $(n=446)$, and through face-to-face interviews with informal carers $(n=7)$ and pwd $(n=5)$. Next, we set up a working group ( $n=15$ ) consisting out of nurses, nurse practitioners, a caregivers, a psychologist, an elderly care physician and a representative of the Dutch Alzheimer's Association to support us with the development of the tools. Besides scientific literature, their practical experience form the basis of the tools. Finally, a field-test was conducted, in which nursing staff $(n=45)$ used the developed tools for three month in daily practice, to help improve the tools even more and learn about enhancing and hampering factors in using them. All this is done to match the tools with the needs in practice as good as possible.

Results Identification of the bottlenecks showed that for person centered care, not only communication between nursing staff and pwd themselves needed to improve, but also between nursing staff and the informal carers and other involved professionals. Therefore, the tools serve as a handle for enhancing communication with all three groups. A guide incorporates recommendations how to communicate within these groups. It is specified whether the recommendations are based on literature, or practical experience by the working group. The recommendations are enriched with practical examples, to make them more tangible. Furthermore, reflection tools and short animations are developed to enhance practical use. In this session the steps in development and the tools themselves will be presented.

Perspectives The Dutch professional nurses organization, called V\&VN, is owner of the tools and they will implement them on a nationwide level. 